\title{
Nahrestagung 2018
}

\section{KURZVORTRÄGE}

Benattou Rachida: Zur Schreibfähigkeit im DaF-Unterricht untersucht an ausgewählten Textsorten algerischer Studierender

Olga Dohmann: „Die größeren Probleme sind dann Dinge zu Papier zu bringen [...] das andere ist nicht so das Problem" - Schreiben in der beruflichen Qualifizierung für Erzieherinnen und Erzieher 3

Klara Dreo, Brigitte Römmer-Nossek, Claudia Hackl, Marcela Hubert, Jadpreet Kaur, Frano P. Rismondo und Erika Unterpertinger: Herausforderungen für eine Schreibdidaktik für DAF/ZStudierende: Eine institutionelle Perspektive

Christine Boubakri / Anil Yilmaz: Schreiben im Fachunterricht anhand von Textsorten 5

Carmen Heine: Integrated Problem Decision Reports (IPDR) im DAF-Kontext 7

Joachim Hoefele/ Liana Konstantinidou: Förderung der allgemeinen Schreibkompetenz in der beruflichen Bildung - Prozessorientierte Schreibdidaktik zwischen DaM (Deutsch als Muttersprache) und DaZ (Deutsch als Zweitsprache)

Irena Horvatić Bilić: Schreibkompetenz in DaF im Kontext der kroatischen Staatsmatura

Helena Hradilková / Anke Sennema: Projektunterricht im schulischen DaF-Unterricht: Schreiben als Sprachhandlung

Patrícia Kertes: Schreiben im universitären DaF-Unterricht - zur Rolle und Funktion der Reflexion im Schreibprozess

Magdalena Knappik: Schreiben als soziale Praxis fassen - Potenziale für die Schreibdidaktik und Lehrendenbildung

Magdalena Michalak / Evelyn Beck: „Die Schüler des 21. Jahrhundert (sind) eingeteilt in Jungen und Mädchen...". Schriftliche Auswertung von Grafiken in der Zweitsprache Deutsch

Julia Plainer: Sprechen über Geschriebenes - Schüler/innen-Schreibbegleitung als Möglichkeit der individuellen Schreibförderung in mehrsprachigen Schreibkontexten

Annemarie Saxalber: Lesen und Schreiben als Kernkompetenzen in der LehrerInnenbildung

Ana Stipančević: Effekte extensiven Lesens und Hörens auf die Entwicklung der Schreibkompetenz im DaF-unterricht - Ergebnisse einer experimentellen Untersuchung

Clara Thöne: Basale Schreibkompetenzen bei neu zugewanderten Kindern und Jugendlichen

Jutta Wolfrum: Schreibförderung in mehrsprachigen Lernergruppen unter Berücksichtigung der Herkunftssprachen 


\section{Rachida Benattou: Zur Schreibfähigkeit im DaF-Unterricht untersucht an ausgewählten Textsorten algerischer Studierender}

Das Verfassen von schriftlichen Texten in einer fremden Sprache ist eine komplizierte und anspruchsvolle Tätigkeit für nicht-muttersprachliche Schreibende, da sie einer besonderen geistigen Leistung bedarf, die auf der Beherrschung vieler Regeln und Normen beruht, im Gegensatz zur mündlichen Produktion.

Neben der Förderung mündlicher kommunikativer Fertigkeiten im algerischen Hochschullehrplan für Deutsch als Fremdsprache wird dem schriftlichen Ausdruck ein große Augenmerk geschenkt.

Der vorliegende Beitrag widmet sich ganz gezielt den Fragen, die mit der Herstellung von Textsorten unter Berücksichtigung von $\mathrm{DaF}$ an der Universität Algier 2 zu tun haben.

Hauptanliegen dabei ist es zu untersuchen, was unter Textsorten zu verstehen ist, was für wesentliche Merkmale sie aufweisen, was für Funktion sie erfüllen und welche Textsorten für welche Zielgruppen konzipiert werden müssen.

Grundlage für diese empirische Untersuchung werden Texte aus der schriftlichen Klausur von Lernenden im dritten und vierten Halbjahr an der Deutschabteilung der Universität Algier 2 herangezogen.

Beim Verfassen dieser Texte sind die Studenten mit vielen Schwierigkeiten konfrontiert, die einen negativen Einfluss auf die qualitative Textproduktion ausüben. Diese Schwierigkeiten beziehen sich hauptsächlich auf die Teilprozesse des Schreibens. Hinzu kommen lexikalische, morphosyntaktische Probleme sowie Probleme mit der Rechtschreibung und Zeichensetzung. Zielsetzung dieser Forschungsarbeit ist es, nach den Ursachen dieser Schwächen und den häufigen sprachlichen Fehlern zu fragen, um diese Schreibprobleme für Studierende zu überwinden.

\section{Zur Person:}

Rachida Benattou, geboren 1957 in Ain-Fekan, Mascara - Algerien

Magister in DaF-Didaktik 2001, Lehrtätigkeit seit 2001

Seit 2011 Dozentin und Lehrerin an der Universität Algier 2, Fachbereich DaF Didaktik, Methodik und Pädagogik, lebt in Algerien

Publikationen im In-und Ausland

Mitglied und Vize Präsidentin des Algerischen Germanisten- und Deutschlehrerverbandes (AGDV) 


\section{Olga Dohmann: „Die größeren Probleme sind dann Dinge zu Papier zu bringen [...] das andere ist nicht so das Problem" - Schreiben in der beruf- lichen Qualifizierung für Erzieherinnen und Erzieher}

An beruflicher Aus- und Weiterbildung zur Erzieherin/ zum Erzieher nehmen vermehrt Personen mit anderen Erst- bzw. Familiensprachen als Deutsch teil. Lehrende sehen sich dadurch vor Herausforderungen gestellt: Viele Schülerinnen und Schüler bzw. Studierende oder Teilnehmende benötigen Unterstützung hinsichtlich fach- und bildungssprachlicher Aspekte des Deutschen. Als eine besondere Herausforderung wird dabei das Schreiben im Fachunterricht wahrgenommen.

Im Vortrag wird ein Teilprojekt Integriertes Fach- und Sprachlernen in beruflichen (Anpassungs-) Qualifizierung für Erzieherinnen und Erzieher vorgestellt, das vom Arbeitsbereich Deutsch als Zweit- und Fremdsprache des Instituts für Germanistik und Vergleichende Literaturwissenschaft der Fakultät für Kulturwissenschaften der Universität Paderborn durchgeführt und vom Förderprogramm Integration durch Qualifizierung seitens der IQ Fachstelle Berufsbezogenes Deutsch gefördert wird. Das Projekt unterstützt Lehrende u.a. in Bezug auf die Schreibförderung (im Fachunterricht). Es werden die in den Interviews der Bedarfserhebung genannten Schwierigkeiten im Bereich Schreiben analysiert und anschließend die Fördermöglichkeiten vorgestellt, die in Workshops mit den Lehrenden erprobt, diskutiert und seitens der Lehrenden insgesamt als angemessen bewertet wurden.

Des Weiteren werden die ersten Evaluationsergebnisse der im Rahmen des Projekts durchgeführten Prozessbegleitung präsentiert. Diese diente zum einen der Unterstützung beim Transfer der Inhalte aus den Fortbildungen in die Unterrichtspraxis und vertiefte das Thema Schreibförderung. Zum anderen beabsichtigte die Prozessbegleitung Professionalisierung der Lehrenden hinsichtlich der Unterstützung ihrer Lernenden im Rahmen einer Schreibwerkstatt.

\section{Zur Person:}

Olga Dohmann, M. A., ist wissenschaftliche Mitarbeiterin im Arbeitsbereich Deutsch als Zweitsprache, Fremdsprache und Mehrsprachigkeit am Institut für Germanistik und Vergleichende Literaturwissenschaft der Universität Paderborn. Ihre Arbeitsschwerpunkte sind integriertes Fach- und Sprachlernen in der beruflichen Qualifizierung, Unterrichten von neu zugewanderten Schülerinnen und Schülern, Deutsch als Zweitsprache und Sprachförderung im Kontext der Mehrsprachigkeit sowie (wissenschaftliches) Schreiben und Schreibberatung. 


\section{Klara Dreo, Brigitte Römmer-Nossek, Claudia Hackl, Marcela Hubert, Ja- dpreet Kaur, Frano P. Rismondo und Erika Unterpertinger: Herausforde- rungen für eine Schreibdidaktik für DAF/Z-Studierende: Eine institutionel- le Perspektive}

Wissenschafltiches Lesen und Schreiben setzen ein Sprachniveau voraus, das die gesetzlichen Anforderungen an die Sprachkompetenz bei Studienbeginn (B2) übersteigt. Daraus ergibt sich der Bedarf für eine hochschuldidaktische Maßnahme, die sowohl wissenschaftliche Schreibals auch Sprachkompetenzen addressiert. Während DaF/DaZ-Didaktik größtenteils einen Fokus auf den Spracherwerb legt, unterstützt prozessorientierte Schreibdidaktik Studierende in ihren individuellen Schreibprozessen, geht aber von vorhandenen sprachlichen Kompetenzen als Grundvoraussetzung aus.

Ein genauerer Blick auf die potentielle Zielgruppe für hochschuldidaktische Maßnahme, die beide verbindet, zeigt, dass diese äußerst heterogen ist. Wir nehmen im geplanten Vortrag eine Analyse der Rahmenbedingungen der Studierenden sowie der Möglichkeiten des Center for Teaching and Learning (CTL) als Institution vor und versuchen, die Bedürfnisse und Zusammenstellung der Zielgruppe(n) zu erfassen. Die Angebote zum wissenschaftlichen Schreibens für Studierende sind mit dem Bereich $\mathrm{DaF} / \mathrm{DaZ}$ über die Ausbildung studentischer SchreibmentorInnen verzahnt. Die Herausforderung besteht darin, einerseits zu erkennen, welches bestehende Angebot für eine Untergruppe der ,Studierenden mit anderer Erstsprache als Deutsch" geeignet ist und welche zusätzlichen Angebote und Handreichungen entwickelt werden sollten.

Im Wintersemester 2017/18 werden erstmals schreibdidaktische Einzelberatungen mit DaF/DaZ- Kompetenz angeboten und zusätzliche Unterstützungsmaterialen im Rahmen der personellen Möglichkeiten ad hoc eingebunden, bzw. entwickelt. Die Beratungen werden in Protokollen und einer Evaluation erfasst, die zu Semesterende ausgewertet und analysiert werden. Die Ergebnisse sowie Erfahrungen in diesem Testlauf werden als Grundlage für weitere Formate im Zusammenhang mit der Schnittstelle DaF/DaZ-Schreibdidaktik präsentiert.

\section{Zur Person:}

Klara Dreo, M. A. ist Linguistin, arbeitet als Schreibassistentin am Center for Teaching and Learning der Universität Wien und studiert den Master Deutsch als Fremd- und Zweitsprache. Claudia Hackl studiert Lehramt Deutsche Philologie und Informatik an der Universität Wien. Neben ihrer Tätigkeit als Schreibassistentin am Center for Teaching and Learning (CTL) ist sie Tutorin für wissenschaftliches Arbeiten am Institut für Germanistik und arbeitet als studentische Mitarbeiterin eines sprachwissenschaftlichen Digitalisierungsprojekts des Austrian Centre for Digital Humanities (ACDH) an der Österreichischen Akademie der Wissenschaft (ÖAW).

Marcela Hubert, B.A., studiert Konferenzdolmetschen im Master Translation an der Universität Wien und ist als Schreibassistentin am CTL tätig.

Jadpreet Kaur, B.A., arbeitet als Schreibassistentin am Center for Teaching and Learning der Universität Wien und studiert den Master Politikwissenschaften.

Frano P. Rismondo arbeitet als Schreibassistent am Center for Teaching and Learning der Universität Wien und studiert den Bachelor Politikwissenschaften.

Mag.a Brigitte Römmer-Nossek ist Kognitionswissenschaftlerin, leitet das Subteam Schreiben am Center for Teaching and Learning der Universität Wien und promoviert über wissenschaftliches Schreiben als kognitiver Entwicklungsprozess an der Universität Wien.

Erika Unterpretinger, B.A., arbeitet als Schreibassistentin am Center for Teaching and Learning der Universität Wien und studiert den Master Europäische und Vergleichende Literaturwissenschaft. 


\section{Christine Boubakri / Anil Yilmaz: Schreiben im Fachunterricht anhand von Textsorten}

Textsorten bedingen schriftsprachliche Handlungsmuster, diese werden durch sprachliche Mittel umgesetzt (u.a. Textstrukturierungsmerkmale), die aus den jeweiligen fachlichen Textsorten funktional hergeleitet werden können. Aus diesem Grund sind Textsorten ein wichtiges Werkzeug, um mit wiederkehrenden Kommunikationsaufgaben im Fach umzugehen (Becker-Mrotzek \& Böttcher, 2006). Im Forschungsprojekt SchriFT-Schreiben im F achunterricht der Sekundarstufe I unter Einbeziehung des Türkischen (gefördert vom BMBF) wurden Zusammenhänge zwischen Fachwissen, fachlicher und fachübergreifender Schreibkompetenz sowie Hintergrundvariablen (z.B. Migrationshintergrund, sozioökonomischer Status) mithilfe von Strukturgleichungs- und Mehrebenenmodellen analysiert. Dazu wurde eine Querschnittserhebung mit 1718 Schülerinnen und Schülern der siebten und achten Jahrgangsstufen an Gesamtschulen in NRW durchgeführt. Die Analyse erfolgt am Beispiel von schulisch relevanten Textsorten in den Fächern Geschichte, Politik, Technik, Physik, Deutsch sowie im türkischsprachigen Herkunftssprachenunterricht. In der zweiten Förderphase werden basierend auf den Ergebnissen der Querschnittserhebung Unterrichtsinterventionen entwickelt. Auf Grundlage des Genre Cycles (Bereiter, 1980; Enli, 2015; Tuan, 2011) werden sprachlich- kognitive Handlungsmuster (z.B. Beschreiben, Erklären und Begründen) (BeckerMrotzek \& Böttcher, 2006; Redder, Guckelsberger, \& Graßer, 2013), mit Bezug zu ihrer fachlichen Verwendung in Textsorten, gefördert. Die Wirksamkeit der Interventionen wird in einer quasi-experimentellen Feldstudie überprüft. Dabei werden einerseits die Erfolge in den Fächern, andererseits Transfereffekte von der Fachsprache in die Bildungssprache, von der Bildungssprache in die Fachsprache sowie vom Türkischen ins Deutsche, untersucht.

Der Vortrag fasst zentrale Ergebnisse der Querschnittserhebung des abgeschlossenen ersten Projektes zusammen. Diese deuten darauf hin, dass Schreibkompetenz im Fachzusammenhang entwickelt werden sollte. Außerdem werden wissenschaftlich erprobte Unterrichtsentwürfe und Materialien vorgestellt, die zeigen, wie Unterrichtsreihen unter Einbeziehung naturwissenschaftlicher und gesellschaftswissenschaftlicher Arbeitsweisen sprachsensibel gestaltet werden können.

\section{Literatur}

Becker-Mrotzek, M., \& Böttcher, I. (2006). Schreibkompetenz entwickeln und beurteilen. Berlin: Cornelsen Verlag Scriptor.

Bereiter, C. (1980). Development in Writing. In L. W. Gregg, \& E. R. Steinberg (Hrsg.), Cognitive Processes in Writing (S. 73-96). New Jersey: Lawrence Erlbaum Associates .

Enli, L. (2015). Implementing Genre-Based Curriculum Cycle in Teaching Writing in Secondary Scholl Settings. Studies in Literature and Language, Vol. 10, No. 1, 47 - 50.

Redder, A., Guckelsberger, S., \& Graßer, B. (2013). Mündliche Wissensprozessierung und Konnektierung. Sprachliche Handlungsfähigkeit in der Primarstufe. Münster: Waxmann.

Tuan, L. T. (2011). Teaching Writing through Genre-based Approach. Theory and Practice in Language Studies, Vol. 1, No. 11, 1471-1478.

\section{Zur Person:}

Christine Boubakri ist seit November 2014 wissenschaftliche Mitarbeiterin im interdisziplinären Forschungsprojekt SchriFT. Dort arbeitet sie interdisziplinär in der Physikdidaktik und im Bereiches Deutsch als Zweit- und Fremdsprache. Ihre Forschungsschwerpunkte sind die Entwicklung von sprachsensiblen Lehr- und Lernmaterialien, die empirische und statistische Aufarbeitung von Schülertexten bezogen auf die Verbindung von fachlichen und sprachlichen Lernen in der Physik und die sprachsensible Gestaltung naturwissenschaftlichen Unterrichts in der Sekundarstufe I.

Anıl Y1lmaz studiert Technik und Mathematik mit Lehramtsoption für Haupt-, Real-, Sekundar- und Gesamtschulen (M.Ed.) an der Universität Duisburg-Essen. Sie ist seit 2017 wis- 
senschaftliche Mitarbeiterin in dem interdisziplinären BMBF-Forschungsprojekt „Schreiben im Fachunterricht der Sekundarstufe I unter Einbeziehung des Türkischen II (SchriFT II)““. Einer ihrer Forschungsschwerpunkte ist die Sprachbildung im Technikunterricht. 


\section{Carmen Heine: Integrated Problem Decision Reports (IPDR) im DAF- Kontext}

IPDR sind ein von Gile (2004) für die Übersetzerausbildung entwickeltes und für die Schreibdidaktik adaptierbares Werkzeug, mit dem sich Entscheidungsprozesse und Problemlösungsprozesse von Studierenden während der Textproduktion offen legen und im Anschluss in Beratung und Unterrichtssituation diskutieren lassen (Heine/Knorr 2016). Studierende machen sich systematisch während des Textproduktionsprozesses schriftliche Notizen $\mathrm{zu}$ aufgetretenen Problemen oder getroffenen Entscheidungen, um diese in der Nachbereitung $\mathrm{zu}$ diskutieren und damit ihren Textproduktionsprozess zu reflektieren. Lehrende nutzen sie, um global und punktuell in die Besprechung von Schreiberereignissen einzusteigen, da IPDR an schwierigen Textpassagen ansetzen und in den Prozess zoomen. IPDR bilden eine Brücke zwischen Schreibaufgabe, Schreibsituation und Schreibprodukt. Sie dienen meist als persönliche Dokumente der mündlichen Nachbereitung von Schreibaufgaben. Da sie nicht nur als Erinnerungstrigger dienen, sondern auch Einblick in das Reflexionsvermögen von Studierenden geben und Textproduktionsprozesse spiegeln können, sollten IPDR-Aufgabenstellungen neu gedacht und aus dem DAF-Lehr-und Lernkontext heraus der systematischen Analyse zugänglich gemacht werden. Eine Studie mit Drittsemester-BA-Studierenden der Universität Aarhus, bei der Textproduktionsaufgaben der Studierenden (Übersetzungen ins Deutsche, Ersttextproduktion und akademische Textkommentierungen auf Deutsch) mit IPDR verknüpft werden, setzt hier an. Für die meisten Teilnehmer*innen der Datenerhebung (Wintersemester 2017) ist Dänisch die Muttersprache und Deutsch eine Fremdsprache. IPDR-Daten werden erhoben, untersucht und klassifiziert. Der Impulsvortrag stellt IPDR als Unterrichtswerkzeug vor, diskutiert die Wertigkeit schriftlich zugänglicher IPDR aus schreibdidaktischer Sicht und erörtert Adaptierbarkeit und Einsatzmöglichkeiten dieses Werkzeugs.

\section{Zur Person:}

Prof. Dr. Carmen Heine ist an der Universität Aarhus, Dänemark an der School of Communication and Culture im Bereich English Business Communication und Deutsche Wirtschaftskommunikation tätig. Sie unterrichtet neben einsprachiger professioneller Textproduktion (DAF und ELF) Fachübersetzen und webbasierte Kommunikation auf Bachelor- und Masterniveau. Schwerpunkte ihrer Forschungstätigkeit sind die Schreib- und Übersetzungsprozessforschung, hierunter besonders deren didaktische Aspekte. 


\section{Joachim Hoefele/ Liana Konstantinidou: Förderung der allgemeinen Schreibkompetenz in der beruflichen Bildung - Prozessorientierte Schreibdidaktik zwischen DaM (Deutsch als Muttersprache) und DaZ (Deutsch als Zweitsprache)}

Im allgemeinbildenden Sprachunterricht an Berufsfachschulen in der Schweiz ebenso wie in den anderen deutschsprachigen Ländern findet sich ein hoher Anteil an Schüler/innen mit linguistischem Migrationshintergrund. In der Regel werden sie nach Richtlinien und Methoden unterrichtet, die für deutschsprachige Schüler/innen gedacht sind. Fraglich ist jedoch, ob die Konzepte des Muttersprachenunterrichts geeignet sind, die heterogenen sprachlichen Biografien der Schüler/innen angemessen zu berücksichtigen. Daher fokussiert das Projekt auf die Förderung der Schreibkompetenz an Berufsschulen, und zwar nicht bzw. nicht ausschliesslich im Medium der Muttersprachendidaktik, sondern auch der Zweitsprachendidaktik, die im Unterschied zur Muttersprachendidaktik durch eine grössere Explizitheit in Bezug auf die formale Seite der Sprache gekennzeichnet ist.

Von der Projektgruppe wurde ein Rahmenkonzept der prozessorientierten Schreibdidaktik zwischen Deutsch als Mutter- und Deutsch als Zweitsprache entwickelt. Gemeinsam mit Lehrpersonen (Mediatorenkonzept) ist dieses Konzept in ein schreibdidaktisches Curriculum für das erste Lehrjahr umgesetzt worden mit handlungsorientierten Schreibanlässen, Lehr/Lernmaterialien, Anleitungen zum Peer-Feedback und sprachlichen Übungen.

Die Wirkung des Konzepts wurde durch eine kontrollierte Interventionsstudie mit Pre- und Posttests und eine Follow-up-Erhebung in 18 Berufsfachschulklassen (neun Experimentalund neun Kontrollgruppen) evaluiert. Zur Überprüfung der Interventionswirksamkeit wurden neben der Schreibkompetenz auch andere, damit zusammenhängende Merkmale wie Selbstwirksamkeitserwartung, Einstellung zum Schreiben usw. mittels Fragebögen erhoben. Die Ergebnisse zeigen signifikante Verbesserungen der Schreibkompetenz vier Monate nach der Intervention.

Der Vortrag skizziert die zugrundeliegenden theoretischen Konzepte, stellt die entwickelten Lehr/Lernmaterialien vor und präsentiert ausgewählte Ergebnisse der Interventionsstudie.

\section{Zur Person:}

Joachim Hoefele, Prof. Dr. phil., Forschungs- und Arbeitsbereich DaF/DaZ, Language Competence Centre, ZHAW

Liana Konstantinidou, Prof. Dr. phil., Forschungs- und Arbeitsbereich DaF/DaZ, Language Competence Centre, ZHAW 


\section{Irena Horvatić Bilić: Schreibkompetenz in DaF im Kontext der kroatischen Staatsmatura}

Im Beitrag wird die Entwicklung der Schreibkompetenz in der Sekundarstufe II (in Kroatien Klassen neun bis zwölf) vor dem Hintergrund der Leistungen, die im Kontext der kroatischen Staatsmatura in DaF erwartet werden, diskutiert. Einführend werden zwei Leistungsniveaus und die ihnen zugeordneten Lernziele, die von dem Nationalen Zentrum für externe Evaluation definierten sind, dargestellt. Einige Aufgabenbeispiele der Maturaprüfung sowohl für das Grundniveau als auch für das höhere Niveau werden präsentiert, wobei der Frage nachgegangen wird, ob die zu produzierenden Textsorten in realen kommunikative Situationen, sei es im Studium, Berufs- oder Privatleben ihre Anwendung finden.

Der Fokus des Beitrags liegt auf der Frage der Schreibdidaktik, die die Entwicklung der vorgeschriebenen Kompetenzniveaus (Grundniveau der Maturaprüfung = A2 laut GER, höheres Niveau der Maturaprüfung = B2 laut GER) entsprechend unterstützen sollte. Als Faktoren, die sich auf die schreibdidaktischen Ansätze auswirken können, werden Aufgaben zur Förderung der Schreibkompetenz in Lehrwerken diskutiert und die Einstellung der DaF-Lehrkräfte dazu, wie wichtig ist die Förderung der Fertigkeit Schreiben im Vergleich zu den anderen drei Fertigkeiten: Sprechen, Lesen, Hören. Die Daten zur Einstellung der Lehrkräfte werden durch einen Fragebogen erhoben. Das Hauptziel der empirischen Datenerhebung ist zu erfahren, welche Praktiken die Lehrkräfte in der Förderung der Schreibkompetenz ihrer SchülerInnen schon anwenden und in welchen Segmenten brauchen sie selber als Lehrkräfte Unterstützung, damit das Endziel der kompetenten schriftlichen Kommunikation im Leben und nicht nur im Kontext der Maturaprüfung in DaF erreicht wird.

\section{Zur Person:}

Dr. Irena Horvatić Bilić ist Lehrende der Abteilung für Fachsprachen der Juristischen Fakultät der Universität Zagreb, wo sie Deutsch und Englisch als Fachsprachen für angehende Juristen und Verwaltungsreferenten unterrichtet. Seit Jahren ist sie auch im DaF-Lektorat eines der größten kroatischen Verlage tätig. Sie ist Autorin einer Lehrwerkserie für Deutsch als zweite Fremdsprache auf dem mittelschulischen Niveau - zweite.sprache@DEUTSCH.de.

Ihre Forschungsinteressen umfassen Mehrsprachigkeitskompetenz und Tertiärsprachendidaktik sowie Fachsprachen und Fachsprachendidaktik. 


\section{Helena Hradilková / Anke Sennema: Projektunterricht im schulischen DaF-Unterricht: Schreiben als Sprachhandlung}

Für tschechische SchülerInnen ist die Bedeutung von Deutsch als Mehrwert für die berufliche Perspektive oft noch abstrakt und erscheint für ihre aktuellen Interessen und Bedürfnisse wenig lebensnah. Im Rahmen einer Projektwoche, die von tschechischen und österreichischen DaF-Studierenden vorbereitet und an einem Gymnasium in Tschechien durchgeführt wird, gehen die SchülerInnen während einer Woche auf die Suche nach Orten in ihrer Stadt, in denen im beruflichen Alltag Deutsch gebraucht und gesprochen wird. Bei Besuchen am Arbeitsplatz gehen sie mit Hilfe von Interviews nach, in welchen beruflichen Zusammenhängen Deutsch in welcher Form gebraucht wird, welche Anforderungen sich für die Beteiligten ergeben und sie reflektieren mögliche eigene Berührungspunkte.

In rhythmisierten Phasen wechselt die Projektarbeit zwischen mündlicher und schriftlicher Modalität (schriftliche Vorbereitung/Dokumentation/Reflexion, mündliche Interviews/Aushandeln der Arbeitsschritte/Reflexion). Im Hinblick auf das Tagungsthema gehen wir den Fragen nach:

Wie gestaltet sich der Wechsel von mündlichen Formen, wie z.B. Interviewsituation oder das Aushandeln von Arbeitsschritten in der Gruppe, und schriftlichen Formen (Interviewleitfaden, Dokumentation, Endprodukte für die Abschlusspräsentation) und welchen Einfluss hat dies auf die Schreibprozesse?

Wo und wie treten Phänomene der Mehrsprachigkeit in den schriftlichen Produkten auf?

Der Beitrag richtet sich an Lehrende in der Sekundarstufe und an Lehrende und Studierende an Hochschulen.

\section{Zur Person:}

Anke Sennema ist Mitarbeiterin im Fachbereich Deutsch als Fremd- und Zweitsprache der Universität Wien.

Helena Hradilková arbeitet als DaF-Lektorin an der Masaryk Universität Brno und unterrichtet Deutsch am Gymnasium in Zastávka. 


\section{Patrícia Kertes: Schreiben im universitären DaF-Unterricht - zur Rolle und Funktion der Reflexion im Schreibprozess}

Der universitäre DaF-Unterricht für Germanistikstudierende hat eine doppelte Aufgabe, zum einen die fremdsprachliche Handlungsfähigkeit, zum anderen die Fähigkeit zum Umgang mit der Wissenschaftssprache zu fördern. Um dieser doppelten Herausforderung gerecht werden zu können, soll der Bewusstheit sowohl bei rezeptiven als auch bei produktiven Aktivitäten eine zentrale Rolle zugewiesen werden. Zum bewussten Umgang mit dem Schreiben kann die Reflexion über die einzelnen Arbeitsschritte in einem prozessorientiert gestalteten Unterrichtsablauf beitragen. Im Beitrag wird der Frage nachgegangen, welche Möglichkeiten Lehrenden und Lernenden zur Verfügung stehen, die einzelnen Schritte des Schreibens zu reflektieren und dadurch den Lehr- und Lernprozess zu effektivieren. Zum einen wird aufgezeigt, wie Lernende die einzelnen Arbeitsphasen des Schreibens beim Planen, Formulieren und Überarbeiten selbstständig und durch einen gegenseitigen Austausch in der Lerngruppe reflektieren können. Zum anderen fokussiert der Beitrag darauf, was der reflektierte Schreibprozess für diese spezifische Zielgruppe mit sich bringen kann. Durch den reflektierten Umgang mit der Sprache des eigenen Textes bildet sich nämlich eine Basis für den strukturierten Sprachgebrauch, die als Grundlage für die Wissenschaftssprache gilt. Darüber hinaus führen Bewusstmachung und Reflexion der einzelnen Schreibschritte zur Herausbildung solcher Lern-, Denk- und Problemlösestrategien, die für das ganze Studium relevant und fachübergreifend verwendbar sind.

\section{Zur Person:}

Dr. Patrícia Kertes

wissenschaftliche Assistentin am Lehrstuhl für Sprachpraxis und Fachdidaktik des Germanistischen Instituts der Eötvös-Loránd-Universität Budapest;

Akademische Laufbahn: Studium der Germanistik und Hungarologie an der Eötvös-LorándUniversität, Promotion mit einer Dissertation über Textproduktion in der Muttersprache textlinguistische Analyse von argumentativen Abiturtexten

Forschungsinteresse: Textproduktion in der Muttersprache und Fremdsprache; textsortenspezifische Merkmale von argumentativen Textsorten; Rolle und Funktion der metapragmatischen Bewusstheit in der Textproduktion; autonomes Fremdsprachenlernen, Sprachbewusstheit und Sprachlernbewusstheit

Lehrtätigkeit: Seminare für Germanistikstudierende im Bereich Sprachpraxis; Entwicklung von Unterrichts- und Prüfungsmaterialien 


\section{Magdalena Knappik: Schreiben als soziale Praxis fassen - Potenziale für die Schreibdidaktik und Lehrendenbildung}

Neben schreibproduktorientierter und schreibprozessorientierter Forschung gibt es auch eine sich zunehmend etablierende Forschungsrichtung, die Schreiben als soziale Praxis betrachtet. Dies bedeutet, den Gegenstand Schreiben weiter als bisher zu fassen: Neben dem Schreibprozess und dem entstehenden Produkt sind in diesem Zugang auch die Schreibenden selbst in besonderer Weise von Interesse, die (machtförmigen) Beziehungen, innerhalb derer sie schreiben - die sie durch ihr Schreiben beeinflussen und die ihr Schreiben beeinflussen -, ihre Positionierungen, sowie die Praktiken, in denen sich das Schreiben vollzieht, und das bedeutet immer: eine besondere Beachtung von schreibenden, aber niemals „,nur“ schreibenden Körpern und Artefakten (Reckwitz 2003). Nachdem es im englischsprachigen Diskurs mit den New Literacy Studies und den Academic Literacies hierzu bereits eine breitere Basis gibt (u.a. Street 1995, Barton/Hamilton/Ivanic 2000, Gee 2000, Lillis 2001), sind in den letzten Jahren auch im deutschsprachigen Diskurs einige Arbeiten entstanden, die den Gegenstand Schreiben in dieser erweiterten Form fassen (Waggershauser 2015, Sturm/Weder 2016, Knappik 2017/in Vorb. 2018). Dieser deutlich erweiterte Gegenstand hat, so die in diesem Vortrag vertretene These, großes Potenzial für die Schreibdidaktik und die Bildung von Lehrenden, die Schreiben unterrichten. Der Vortrag gibt einen Einblick in das Anliegen dieser Forschungsrichtung, erläutert deren theoretische Bezüge und formuliert anhand konkreter Beispiele aus dem DaZ-Kontext, welches Potenzial durch diese Forschungsrichtung für die Schreibdidaktik entsteht.

\section{Literatur:}

Barton, David; Hamilton, Mary; Ivanič, Roz (Hg.) (2000): Situated literacies. Reading and writing in context. London, New York: Routledge (Literacies).

Gee, James Paul (2000): The New Literacy Studies: from 'socially situated' to the work of the social. In: David Barton, Mary Hamilton und Roz Ivanič (Hg.): Situated literacies. Reading and writing in context. London, New York: Routledge (Literacies), S. 180-196.

Knappik, Magdalena (2017): Schreiben für Viabilität. Konstitutionsprozesse von SchreibendenSubjekten in der Migrationsgesellschaft. Universität Wien: Dissertation (Print-Publikation in Vorbereitung).

Lillis, Theresa M. (2001): Student writing. Access, regulation, desire. London: Routledge (Literacies).

Street, Brian V. (1995): Social literacies. Critical approaches to literacy in development, ethnography and education. London: Longman (Real language series).

Sturm, Afra; Weder, Mirjam (2016): Schreibkompetenz, Schreibmotivation, Schreibförderung. Grundlagen und Modelle zum Schreiben als soziale Praxis. Seelze: Klett/Kallmeyer (Reihe Lehren lernen - Basiswissen für die Lehrerinnen- und Lehrerbildung).

Waggershauser, Elena (2015): Schreiben als soziale Praxis. Eine ethnographische Untersuchung erwachsener russischsprachiger Zweitschriftlernender. Tübingen: Stauffenburg-Verlag (Deutsch als Fremd- und Zweitsprache, Band 6).

\section{Zur Person:}

Magdalena Knappik, Senior Scientist am Fachbereich Deutsch als Fremd- und Zweitsprache der Universität Wien. Promotion zur Entwicklung von Schreibenden in der Migrationsgesellschaft anhand der Analyse von Schreiber*innenbiographien. Arbeits- und Forschungsinteressen: Schreiben als soziale Praxis, (wissenschaftliche) Schreibdidaktik; sprachliche Bildung und Sprachförderung im Kontext Schule; Sprachaneignung im Kontext von Zwei- und Mehrsprachigkeit; gesellschaftstheoretische Fundierung des Felds Deutsch als Zweitsprache, insbesondere mit Bezug auf Rassismuskritik, Migrationspädagogik und Postkoloniale Theorie; ethnographische Methodologie und Praxistheorie. 


\section{Magdalena Michalak / Evelyn Beck: „Die Schüler des 21. Jahrhundert (sind) eingeteilt in Jungen und Mädchen...“. Schriftliche Auswertung von Grafiken in der Zweitsprache Deutsch}

Für die Betrachtung von komplexen Sachverhalten werden im Fachunterricht Diagramme oder Grafiken eingesetzt, die Informationen stark verdichtet präsentieren. Die SchülerInnen stehen vor der Herausforderung, derartige nichtlineare Darstellungsformen im fachlichen Kontext zu erfassen und die erschlossenen Informationen schriftlich (oder auch mündlich) zu versprachlichen. Vereinzelte Studien zeigen, dass Lernende mit Deutsch als Zweitsprache bei diesem Transfer von einer bildlichen Form zu einem Text unsicher sind. Bei der Grafikauswertung bleiben sie meist auf der beschreibenden Ebene; sie verfügen nur bedingt über Diagrammschemata und diagrammspezifische sprachliche Routinen (Michalak et al. 2017).

Im Hinblick auf die Förderung der Schreibkompetenz bei SchülerInnen mit DaZ stellt sich die Frage, wie sich ihre Sprachkompetenz auf die schriftliche Analyse einer Grafik auswirkt und welche Aufgaben sich für die Hinführung der SchülerInnen an Grafiken besonders gut eignen. Bislang ist außerdem ungeklärt, wie die schriftliche Analyse einer Grafik aus fachlichinhaltlicher und sprachlicher Sicht beurteilt werden kann. An diesem Desiderat setzt das Projekt GraFau - Grafiken im Fachunterrcht an. Hierfür wurden 1000 Texte (schriftliche Analyse einer Grafik) von Schülern an Mittelschulen im Fach GSE (Geschichte/Sozialkunde/Erdkunde) sowie deren Sprachkompetenz erhoben. Die Texte wurden mit einem neu entwickelten Kriterienkatalog ausgewertet, der diagrammspezifisch-fachliche, methodische und sprachliche Aspekte berücksichtigt. Ausgehend von den in den Aufgabenstellungen geforderten Sprachhandlungen und den bisherigen Ergebnissen im Projekt werden in dem Beitrag Faktoren analysiert, die im Schreibprozess beim Umgang mit Grafiken ausschlaggebend sind. Auf Grundlage der Auswertung werden didaktische Implikationen formuliert.

Literatur: Michalak, M.; Lemke, V.; Kölzer, C. (2017): „Wenn ich hingucke, seh ich immer erst das Obere“. Kompetenzen von Lernenden mit Deutsch als Zweitsprache beim Umgang mit diskontinuierlichen Darstellungsformen. In: Fuchs, I.; Jeuk, S.; Knapp, W. (Hrsg.): Mehrsprachigkeit: Spracherwerb, Unterrichtsprozesse, Schulentwicklung. Stuttgart: Fillibach bei Klett, S. 73-90.

\section{Zur Person:}

Prof. Dr. Magdalena Michalak ist Inhaberin des Lehrstuhls Didaktik des Deutschen als Zweitsprache an der Friedrich-Alexander-Universität Erlangen-Nürnberg. Ihre Forschungsschwerpunkte liegen u.a. im Bereich des Zweitsprachenerwerbs und der Zweitsprachdidaktik, der Sprachförderung von Kindern und Erwachsenen mit Migrationshintergrund und dem sprachsensiblen Unterricht.

Evelyn Beck ist Akademische Rätin am Lehrstuhl DiDaZ an der FAU. Gemeinsam arbeiten sie derzeit am Forschungsprojekt „Fachlicher und sprachlicher Umgang von Schülern mit deutscher und nichtdeutscher Erstsprache mit Grafiken im Unterricht"“. 


\section{Julia Plainer: Sprechen über Geschriebenes - Schüler/innen- Schreibbegleitung als Möglichkeit der individuellen Schreibförderung in mehrsprachigen Schreibkontexten}

In dem Impulsvortrag wird im Kontext von mehrsprachigen Schreibsituationen eine Schüler/innen-Schreibbegleitung (Roll 2012) zur Erweiterung von Schreibkompetenz vorgestellt, die in kreativen- und biographischen Schreibwerkstätten des Ruhrgebiets entwickelt wurde und im binationalen INTERREG-Austauschprojekt „Nachbarsprache \& Buurcultuur“ erprobt wird.

Um mehrsprachige Schreibsituationen zu entlasten, werden Schüler/innen zu schreibbegleitenden „Peer-Paten“ ausgebildet. In der Ausbildung erwerben die angehenden Schreibbegleitenden unter anderem Basiswissen zu Planungs- und Schreibstrategien, Schreibblockaden, sprachfördernden (Schreib-) Spielen sowie Feedbackmethoden. Gleichzeitig reflektieren sie ihren eigenen Schreibprozess und vollziehen einen Perspektivwechsel von Schreibenden zu Beratenden. Anhand von selbstverfassten Texten führen sie erste Beratungsgespräche durch und üben die nicht-direktive Beratungsmethode (Bräuer 2007) ein.

Je nach Schreibsituation schreiben und beraten die Schreibbegleitenden als „Peer-Paten“ eine festgelegte Anzahl von Schüler/innen im schulischen Ganztag beziehungsweise andere Schüler/innen in einer Schüleraustauschsituation.

Der Fokus der Schreibbegleitung liegt auf der makrostrukturellen Überarbeitung von Textprodukten, da diese im regulären Unterricht häufig zu wenig Raum findet. Durch die in der Schreibbegleitung festgelegten Schreib- und Überarbeitungsziele können die Schüler/innen schrittweise an einzelnen strukturellen Aspekten des Textes arbeiten. Neben der Schreibbegleitung haben die „Peer-Paten“ selbst ausreichend Zeit, um an eigenen Textprodukten zu arbeiten.

Das Konzept der „Peer-Paten-Begleitung“ soll einen Impuls für den Umgang mit schulischer Heterogenität geben, indem es Ideen für eine intensivere Schreibberatung vorlegt, durch die die individuelle Förderung der Schreibenden zusätzlich unterstützt wird.

\section{Zur Person:}

Julia Plainer beschäftigt sich in ihrem Dissertationsvorhaben mit mehrsprachigen Schreibberatungsgesprächen im Kontext von binationalen Schüleraustauschprojekten. Sie arbeitet als wissenschaftliche Mitarbeiterin von Frau Prof. Dr. Heike Roll am Institut für Deutsch als Zweit- und Fremdsprache der Universität Duisburg-Essen und im Monitoring des INTERREG-Projektes „Nachbarsprache \& Buurcultuur“. Sie arbeitete in der Weiterbildung von Lehrkräften, die mit neu Zugewanderten arbeiten und leitete kreative Schreibwerkstätten an unterschiedlichen Schulformen und Standorten im Ruhrgebiet. Ihre Examensarbeit verfasste sie zum Thema „Mehrsprachige Schülerinnen und Schüler schreiben Slam Poetry unter Begleitung von Studierenden - eine exemplarische Analyse von Textversionen und narrativen Schülerinterviews“". 


\section{Annemarie Saxalber: Lesen und Schreiben als Kernkompetenzen in der LehrerInnenbildung}

Die Ausbildung der KindergärtnerInnen und GrundschullehrerInnen an der Freien Universität Bozen (FUB) hat im überarbeiteten Studienplan den Anteil an Lehrveranstaltungen in der Zweitsprache Deutsch oder Italienisch und in Englisch verstärkt. Dies verlangt von den Studierenden ein effizientes Arbeitsverhalten und einen gekonnten Umgang mit verschiedenen Fachsprachen und -kulturen. Dieser Anspruch wird den Studierenden erleichtert, wenn auch die DozentInnen eines Studienganges bereit sind, den eigenen Habitus in der Kommunikation zu reflektieren und die Lehrdidaktik zu diskutieren. Angepeilt werden soll eine Wissenschaftskultur, die sich die Auseinandersetzung mit anderen Wissenschaftskulturen dezidiert zur Aufgabe macht.

Unter anderem geht es auch um ein Sichverständigen über grundlegende transversale Fertigkeiten im Rahmen von CALP (Cognitive Academic Language Proficiency), deren bewusster Einsatz den Sprachhandlungen dem Ausbau der (akademischen) Lese- und Schreibkompetenzen in L1, L2, wie L2 zugute kommt. Auf dieser Basis können methodische Werkzeuge bereitgestellt werden, die das selbstständige oder kollaborative Lösen von disziplinären Aufgaben fördern. Auch soll erreicht werden, dass die Studierenden Ressourcen, die sie aus anderen Sprachen oder anderen Lehrkulturen ziehen können, gezielt nutzen lernen.

Im Beitrag wird Folgendes angestrebt:

- ein Bild zu geben über die Schreibkompetenzen der StudentInnen zu Studienbeginn (FUB); ausgewählt werden dabei solche Aspekte, die für das wissenschaftliche Lesen Schreiben in Deutsch/L1 wie in Italienisch/L2 von Bedeutung sind;

- Lese- und Schreibaufgaben zu modellieren, die den Studierenden helfen, transversale Kompetenzen zu nutzen;

- an Beispielen von Lehrveranstaltungen zu diskutieren, wie DozentInnen, TutorInnen und SchreiberaterInnen einem prozessorientierten und lernstrategischen Zugang zu Lehrinhalten mehr Gewicht geben können.

\section{Zur Person:}

Annemarie Saxalber, Dr., seit 2010 Prof.in - Deutsche Sprache und ihre Didaktik an der Fakultät für Bildungswissenschaften an der Freien Universität Bozen (I), 2012-2104 wissenschaftliche Leiterin des Schreibzentrums an der Fakultät; von 2007 - 2010 Prof.in am Kompetenzzentrum für Deutschdidaktik an der Alpen-Adria-Universität Klagenfurt (A), dort mitverantwortlich für wissenschaftliche Konzeption der Standardisierten Reife- und Diplomprüfung Deutsch (SRDP). Forschungsschwerpunkte: Forschung zu Schreibdidaktik (sekundäre und tertiäre Bildungsstufe), Bildungssprache, Integrierte Sprachendidaktik. 


\section{Ana Stipančević: Effekte extensiven Lesens und Hörens auf die Entwick- lung der Schreibkompetenz im DaF-Unterricht - Ergebnisse einer experi- mentellen Untersuchung}

Im vorliegenden Beitrag wird die Bedeutung extensiven Lesens und Hörens im DaFUnterricht herausgestellt sowie Auswirkungen dieser Aktivitäten auf die Entwicklung der Schreibkompetenz.

Der Ansatz des extensiven Lesens und Hörens baut auf der Input-Hypothese von Krashen auf, der die These vertritt, dass ein massiver Kontakt mit der Sprache rezeptive und produktive Fähigkeiten steigert. Bezüglich des Ansatzes des extensiven Lesens und Hörens in der Fremdsprache bestehen jedoch Zweifel, ob Lesen und Hören allein tatsächlich zur Steigerung der Lese-, Hör- und Schreibkompetenz führen, oder ob sie durch zusätzliche Aktivitäten ergänzt werden sollten. Laut der Output-Hypothese muss der Input durch aktive Sprachverarbeitung ergänzt werden, um den Spracherwerbsprozess voranzutreiben.

Ein Großteil der extensiven Lernprogramme findet seine Anwendung im asiatischen Raum im Bereich der Erwachsenenbildung, während es im deutschen Sprachraum, vor allem auch im DaF-Bereich, kaum empirische Untersuchungen gibt. In diesem Beitrag werden Ergebnisse einer solchen empirischen Studie dargestellt, die 2014/15 an der Philosophischen Fakultät in Novi Sad (Serbien) mit Studierenden des Wahlfachs Deutsch (Niveaustufe A2) durchgeführt wurde. Während in der Experimentalgruppe extensive Lernprogramme angewandt wurden, wurde der Kontrollgruppe regulärer Unterricht erteilt, im Rahmen dessen verschiedene Lehrwerke und eine Vielfalt von didaktischen Materialien eingesetzt wurden. Die Dauer der Untersuchung betrug zwei Semester, in Folge deren die Lernenden in Hinblick auf ihre Schreibkompetenz getestet wurden. Die Daten wurden quantitativ ausgewertet, während die Gruppenunterschiede mithilfe des t-Tests statistisch untersucht wurden. Die Ergebnisse der Untersuchungen weisen darauf hin, dass sich extensive Lese- und Hörprogramme positiv auf die Schreibkompetenz auswirken und zwar vor allem dann, wenn Input mit Output verbunden wird, bzw. wenn Input und Output durch schriftliche und mündliche Aktivitäten ergänzt werden.

Schlüsselwörter: Extensives Lesen und Hören, Input- und Output-Hypothese, Schreibkompetenz, experimentelles Untersuchungsdesign, DaF-Unterricht.

\section{Zur Person:}

Dr. Ana Stipančević ist Dozentin für die Deutsche Sprache und ihre Didaktik am Institut für Germanistik der Philosophischen Fakultät der Universität Novi Sad (Serbien). 2016 hat sie zum Thema „Effekte der Anwendung von authentischen Texten im DaF-Unterricht“ promoviert. Sie hält verschiedene Lehrveranstaltungen im Bereich von Metodik und Didaktik des DaF-Unterrichts im Bachelor- und Masterstudiengang am Institut für Germanistik der Philosophischen Fakultät der Universität Novi Sad. Sie ist auch in der Praxis tätig und gibt seit 2008 DaF-Kurse. 


\section{Clara Thöne: Basale Schreibkompetenzen bei neu zugewanderten Kindern und Jugendlichen}

Kindern und Jugendlichen, die jenseits des Grundschulalters ins deutsche Bildungssystem einsteigen und über wenig bis gar keine Deutschkompetenzen verfügen, steht nur wenig Zeit zur Verfügung, um sowohl basale Deutschkenntnisse als auch die komplexen bildungssprachlichen Strukturen des Deutschen zu erwerben. Anders als Kinder im Grundschulalter werden sie bei der (auf den Vorbereitungsunterricht folgenden) Eingliederung in den Regelklassenunterricht sofort mit einer konzeptionell schriftlichen Sprachvarietät sowie fachsprachlichen Varianten in allen unterrichtlichen Belangen konfrontiert. Um erfolgreich am Unterricht teilnehmen zu können, müssen sie in kurzer Zeit entsprechende Sprachkompetenzen erwerben.

Dem Schreiben als literaler Teilkompetenz kommt im schulischen Lernen eine bedeutsame Rolle zu. Basale Schreibfähigkeiten, zu denen neben handschriftlichen auch orthografische und grammatische Kompetenzen sowie ein effizienter Zugang zum mentalen Lexikon gehören, werden in der Sekundarstufe als erworben vorausgesetzt, sind jedoch bei neu zugewanderten Kindern und Jugendlichen zu Beginn ihres zweitsprachlichen Erwerbs nicht umfassend ausgebildet. Ihre Bedeutung für den Schreibprozess besteht in der möglichen und nötigen Automatisierung einzelner Teilprozesse und der damit verbundenen Freisetzung kognitiver Ressourcen für hierarchiehöhere Prozesse, deren Beherrschung wiederum die Textqualität wesentlich beeinflusst.

Der Vortrag greift Erkenntnisse des gegenwärtigen Forschungsstands zu erst- und zweitsprachlichen Schreibprozessen und Schreibentwicklungen sowohl kindlicher wie jugendlicher Schreiber*innen auf. Insbesondere wird folgenden Fragen nachgegangen:

- Wie) Unterscheidet sich das Schreiben in der L2 vom Schreiben in der L1? Interagieren jeweils dieselben Teilprozesse?

- Lassen sich die gleichen oder ähnliche Prädiktoren für Schreibleistungen bestimmen, wie sie für das L1-Schreiben angenommen werden?

Das Ziel ist es, aus der schmalen empirischen Evidenz diejenigen Aspekte herauszuarbeiten, auf die im Hinblick auf die Entwicklung und Förderung basaler Schreibkompetenzen bereits im Anfangsunterricht der Vorbereitungs- bzw. Willkommensklassen besonderes Gewicht gelegt werden sollte.

\section{Zur Person:}

Clara Thöne, M.A. Deutsch als Fremdsprache, seit Sommersemester 2014 Lehrkraft für besondere Aufgaben an der Humboldt-Universität zu Berlin, Professional School of Education, im Fachbereich Sprachbildung/Deutsch als Zweitsprache. Der Schwerpunkt meiner Tätigkeit liegt innerhalb der Lehramtsausbildung im Modul Sprachbildung/DaZ für Studierende aller Lehramtsfächer; Ziel ist das Vermitteln von Kenntnissen zum sprachsensiblen Fachunterricht. Lehraufträge an der FU Berlin sowie am Institut für deutsche Sprache und Linguistik der HU Berlin. 


\section{Jutta Wolfrum: Schreibförderung in mehrsprachigen Lernergruppen un- ter Berücksichtigung der Herkunftssprachen}

Betrachtet man die sprachlichen Ressourcen von Schülern und Schülerinnen durchschnittlicher Volks und Mittelschulklassen, so drängt es sich geradezu auf, diese - entgegen dem monolingualen Habitus - für eine effektive Schreibförderung zu nutzen.

Die Herkunftssprachen können dabei in den verschiedenen Phasen des Schreibprozesses berücksichtigt werden, sowohl in der Vorentlastung, der (kollektiven) Planung, der Textproduktion als auch im Rahmen mehrsprachiger Diskurse während der Überarbeitung.

Der Einbezug der Herkunftssprachen ist besonders für Seiteneinsteiger eine große Hilfe, da fehlende sprachliche Strukturen gemeinsam erarbeitet und dadurch der großen Herausforderung des Schreibens in der Zweitsprache mit mehr Selbstbewusstsein begegnet werden kann. Notwendige Differenzierungen ergeben sich dabei ungezwungen, da die Lernenden sich mit ihren Stärken und Schwächen einbringen. Besonders für neu Zugewanderte ist dieses Vorgehen von Vorteil, da sie ihr Weltwissen einbringen können und nicht - wie üblich - an sprachlichen Hürden scheitern müssen.

In anschließenden reflexiven Phasen können Unterschiede hinsichtlich der Textsortenkonventionen in den Herkunftsländern, oder auch Gemeinsamkeiten und Unterschiede auf syntaktischer Ebene bewusst herausgestellt werden, was sowohl für DaM als auch für DaZ-Lernende bereichernd ist, da Erkenntnisse über die eigene(n) Sprache(n) gewonnen werden. Dabei hat sich der Einsatz von Wortartensymbolen nach M. Montessori als zielführend erwiesen, weshalb dieser an Hand von kontrastiven Beispielen aufgezeigt wird.

Zielgruppe: Lehrende in der Sekundarschule

\section{Zur Person:}

Prof. (Univ.Thess.) Dr. Jutta Wolfrum

Derzeit akademische Rätin am Lehrstuhl für Didaktik der Deutschen Sprache und Literatur, Otto-Friedrich-Universität Bamberg; Studiengangsbeauftragte für die Didaktik des Deutschen als Zweitsprache

Bis 2015: DAAD-Lektorin und anschließend Wissenschaftliche Assistentin/Assistemzprofessorin für Angewandte Linguistik an der Aristoteles-Universität Thessaloniki

Forschungsschwerpunkte: Schreibdidaktik, Kreatives Schreiben, Mehrsprachigkeit(sförderung), Kulturgeprägtheit von Textsorten, Zweit- und Fremdsprachendidaktik 